\title{
Autonomous Distributed Energy Management for Intelligent Microgrids
}

\author{
Zeineb Mhadhbi, Cedric Gueguen, Sajeh Zairi, and Belhassen Zouari
}

\begin{abstract}
This paper proposes a new decision approach in energy management systems on a large scale. This approach aims to optimal manage energy demand in real-time by considering share renewable energy excess between microgrids in order to minimize energy consumer costs, maximize energy producer gains and reduce gas emissions. A microgrid, in seller mode, may have a risk of a decreasing profitability when the excess energy is supplied. As advance estimation of energy consumption and production may give an unstable input, so this microgrid may confirm to sell a quantity of energy which will be required later for itself. To overcome this inconvenience, a profitable energy selling price which is based on the energy selling risk is a good solution to avoid inefficient sales. In our solution, profitability is increased thanks to our energy selling price which is defined in a flexible manner and is adequately adapted to the microgrid's excess energy and the profitability factor. Simulation results show that our energy sharing model satisfy microgrid's requirements.
\end{abstract}

Index Terms-Energy management, energy efficiency, cost optimization, gain maximization.

\section{INTRODUCTION}

Today, the rate of consumption from fossil fuel (petroleum, coal and natural gas) is very high [1]. The world depends on fossil fuels to satisfy our energy needs. According to the U.S. Energy Information Administration (EIA) in 2014, 66\% of the nation's electricity was generated by fossil fuels [2]. However, fossil fuels when burnt can lead to have serious environmental problem such as air pollution that leads to global warming (climate change) [1], [3], [4]. The latter is affecting many parts of the world and therefore can have severe consequences on the habitats.

The appearance of new productions from distributed renewable sources which are increased sharply in last decade may allow for decrease our dependence on fossil fuels drastically. Renewable energy sources become an instrument of climate policy. This new intermittent production (distributed generation) must be efficiently integrated into the electricity distribution system [5]. A microgrid is a platform composed of distributed energy resources, distributed energy storage devices and loads which can be disconnect from the traditional grid and operate

Manuscript received September 17, 2016; revised June 12, 2017

Z. Mhadhbi is with the Mediatron Research Laboratory, Higher School of Communications, University of Carthage, 2083, Ariana, Tunisia (e-mail: zaineb.mhadhbi@gmail.com, belhassen.zouari@supcom.tn).

C. Gueguen is with the Institut de Recherche en Informatique et Systèmes Aléatoires Laboratory, University of Rennes1, 35000 Rennes, France (e-mail: cedric.gueguen@irisa.fr).

S. Zairi is with the Higher School of Digital Economy, University of Manouba, 2010 Manouba, Tunisia, and also with the Mediatron Research Laboratory, Higher School of Communications, University of Carthage, 2083, Ariana, Tunisia (e-mail: zairisajeh@fst.rnu.tn). autonomously. Accordingly, an intelligent microgrid appears necessary [5]. Microgrid is taken as the future smart grid. Such smart grid aims to better manage peak electricity demands, optimize the energy transfer cost, integrate easily news electricity sources and uses (Photovoltaic, Electric Cars consumption...) and mostly reduce gas emissions. In smart microgrid, energy management research has recently received considerable attention, as can be seen in the large amount of work reviewed in [5]. However, to motivate consumers to actively and voluntarily participate in such management programs is one of the key challenges of successful energy management [5]-[7]. Furthermore, energy management has become an active topic which aims at one or both of the following design objectives: reduce gas emissions by automatically reducing and shifting consumption in microgrid at peak hours in response to varying electricity price [8]-[10].

Various energy management approaches were developed for smart microgrids like: the Optimization based Residential Energy Management (OREM) [11], in-Home Energy Management (IHEM) [11], Residential Load Control (RLC) [12], Decision support Tool (DsT) [13], Appliance Coordination (ACORD) [14], optimum load management (OLM) [15], Optimal Consumption Schedule (OCS) [16], Appliance Coordination Scheme (ACS) [17], microCHP based Scheduling [18], incentive-driven Distributed Energy Sharing system (iDES) [19], and Multiagent Based Distributed-Energy- Resource Management (multiagent systems (MASs)) [20]. The pricing schemes like, Real Time Pricing (RTP), Time of Use (ToU), Inclining Block Rates (IBR), Critical Peak Pricing (CPP), day ahead pricing (DAP) have been proposed by distribution companies for smart grid in order to lead towards efficient electricity use [21]. Their main goal is encouraging microgrids to shift their load individually and voluntarily in order to reduce their consumption at peak hours.

Some of existing approaches have considered the management of the energy excess locally and at neighborhood (between microgrids/neighbors) [19], [20]. Indeed, sharing energy between neighbors allows for:

- Better profitability for every microgrid $n$, because when it is:

- $\quad$ in seller mode, it may maximize its gains by selling its excess energy to the neighbors at a Selling Price (SPn), which is higher than the grid's Buying Price (BP).

- $\quad$ in buyer mode, it may reduce the energy bill by buying energy from neighbors at a Selling Price (SPn) which is less than the grid's Selling Price (SP).

- Better efficient energy management since local energy purchase makes it possible to satisfy neighbors' requests without needing to shift the 
consumption in microgrid.

Sharing energy between microgrids is based on an estimation of the energy consumption and generation. However, advance estimation may give an unstable (or uncertain) input because consumption and production may increase or decrease over the time. At a given time, a microgrid may act as a seller (i.e. when it has excess energy) and/or a buyer (i.e. when a lack of electric energy takes place) In fact, based on uncertain inputs, the microgrid, in seller mode, may estimate that it will have an excess of electric energy. Therefore, this microgrid will agree to sell an amount of energy to its neighbors. However, sometimes the actual microgrid energy consumption may exceed the predicted consumption or/and the actual microgrid production is lower than the predicted production. In this case, the seller may confirm to sell a quantity of energy which will be required for himself later. Therefore, when the energy will be required later, this microgrid will be obliged to buy energy from its neighbors (other microgrids) or from electric utility companies (grid) with high prices. Selling energy that will be required later conduct to profitability loss for the seller because, in the majority of cases, it will be obliged to buy energy more costly than the energy that it had.

To overcome this sort of inconvenience, we propose in this paper, on a large scale, a real-time decision approach for each microgrid (homes, neighborhoods, cities, institutional buildings, campuses, etc.) called Real-Time Distributed Energy Management (RTDEM). Based on RTDEM approach, a microgrid, in seller mode, must propose a profitable selling price. The defined selling price must be flexible and adaptable to its surplus energy and a profitability factor. This profitability factor is necessary to determine whether the surplus energy proposed for sale will be required later for itself in order to avoid non-optimal sales operations and, consequently, maximize the seller's gain. When the microgrid is in buyer mode, it decreases its energy bill by using its own energy then the energy from the neighbors by choosing the best alternative to satisfy its needs and lastly the energy from the utility companies (grid). The best alternative is determined by solving an optimization problem considering different criteria such as selling price, available energy, etc.

The present work focuses on the validation of the new realtime decision approach on a large scale by considering share renewable energy excess not only between homes but also between neighborhoods, campuses, cities, etc. in order to:

- motivate the local renewable energy investment,

- minimize energy losses from fossil fuels.

In our solution, profitability is increased thanks to our realtime management. This management may be improved based on a gainful energy selling price. RTDEM decreases the energy cost by optimally choosing, in real-time, between local or neighborhood-generated energy. It requests grid-supplied only in case of neighbors'energy shortage. Our proposal also maximizes each producer's gains by selling its excess energy not only to the utility companies but also to neighbors at an optimal selling price, which will motivate the local renewable energy investment.

This paper is organized as follows. Section II is the proposed approach RTDEM. Section III reports simulation results. Section IV concludes the material.

\section{REAL-TIME DistribUtED ENERGY MANAGEMENT}

(RTDEM)

At a given time, each microgrid in our system may act as a seller (when it produces more than it consumes) and/or a buyer (when its local energy source is not sufficient to satisfy its demand). When the microgrid is in buyer mode, it may reduce the energy bill by buying energy from neighbors at a Selling Price (SPn) which is less than the grid's Selling Price (SP). RTDEM enables to satisfy in real-time any demand by requesting at first time local energy, second it requests neighbors in case local energy shortage, and at worst it demands to grid. When the microgrid is in seller mode, it may maximize its gains by selling its excess energy to its neighbors at SPn, which is higher than the grid's Buying Price (BP).

At any time, a microgrid $\mathrm{n}$ may receive a request for energy purchases from the neighbors. Upon receiving the request, the microgrid $\mathrm{n}$ its excess of energy based on an estimation of energy consumption and production for the requested time slot. If there is an excess energy, this seller must maximize its gain by proposing an optimal energy selling price.

In most existing energy management approaches, the excess energy is sold to neighbors at a price that depends on Acceptable Revenue Level (ARL) [19], [20]. ARL is defined as the unit cost of energy. However, this price is difficult to define adequately and may potentially vary for each microgrid which decreases the profitability of the microgrid who has a high ARL. With a high ARL, microgrid cannot sell energy, when the supply $\geqslant$ demand, to neighbors its excess energy.

In addition, cost amortization cannot be compatible with a price that allows selling to neighbors (excess energy price $>$ $\mathrm{SP}$ ). In this case, microgrids will always prefer energy from the company utility that will not motivate the microgrid to actively and voluntarily participate in energy management. In order to promote energy sharing and renewable energy, we propose that the excess energy must be sold at an adequate and adaptive price. It will be more profitable for the seller $n$ sell energy to the neighbors at a selling price ( $\mathrm{SPn})$, which is higher than BP and less than SP, because neighbors trade only with sellers which propose a SPn less than SP.

$$
\mathrm{BP}<\mathrm{SPn}<\mathrm{SP} .
$$

Three main factors that will impact the price SPn which are: supply-demand relationship, excess energy and profitability factor.

- If (Supply $\leqslant$ Demand) then seller proposes a high price:

$$
\mathrm{SPn}=(\mathrm{SP}-\epsilon)
$$

- $\quad$ Else (if Supply > Demand) then SPn depends on the excess energy and the profitability factor.

$$
\mathrm{SPn}=\mathrm{f}(\text { Excess, } \mathrm{pfn})
$$

A rate of profitability factor pfn is necessary for each microgrid $\mathrm{n}$ to specify the Energy Selling Risk (ESRn). Profitability factor may define approximately the probability 
of the use of excess energy later. If $\mathrm{pfn}=0 \%$, the seller don't trust on its estimation and may require its excess energy. Its ESRn is equal to $100 \%$. Otherwise, if $\mathrm{pfn}=100 \%$, then the seller trust on its estimation and is certain that it will not consume its excess energy. Its ESRn is equal to $0 \%$. Profitability factor is calculated according to the historical of the total of previous Right amount Sold (RS) divided by the Total quantity Sold (TotS) for the requested Time Slot (TS).

$$
p f_{n}(\%)=\frac{\sum R S}{\sum T o t S}
$$

The proposed selling price SPn must be less than SP and higher than BP. If seller has a lot of energy and his profitability factor is great (equal to $100 \%$ ), then it wants to flow (sell) a maximum energy to its neighbors since it is more profitable to sell surplus at SPn to neighbors than at BP to utility companies (grid). Thus, it offers a low price:

$$
\mathrm{SPn}=(\mathrm{BP}+\epsilon)
$$

Otherwise, if the seller has less energy and his profitability factor is low (equal to $0 \%$ ), then seller avoids selling excess energy which could be required later for itself by offering a high price:

$$
\mathrm{SPn}=(\mathrm{SP}-\epsilon)
$$

With high price, seller has low chance to sell its energy when the supply is greater than demand. However, if its consumption increases later, its own energy will stay available and avoid buying energy at higher price. In general cases, each seller $\mathrm{n}$ determines, at each request, its energy selling price SPn by using the following function:

$$
\begin{aligned}
& S P_{n}=\frac{\left|p f_{n}-100\right| \times\left(S P_{\max }-\left(S P_{\min }+\left(S P_{\max }-E_{n}\right)\right)\right)}{100} \\
& +\left(S P_{\min }+\left(S P_{\max }-E_{n}\right)\right)
\end{aligned}
$$

$$
\begin{gathered}
E_{n}=\frac{\text { Excess } \times\left(\text { SPmax }-S P_{\min }\right)}{M a x\left(\text { Excess }, \max _{R E}\right)}+S P_{\min } \\
S P_{\max }=S P-\epsilon \\
S P_{\min }=B P+\epsilon \\
S P_{\min } \leqslant E_{n} \leqslant S P_{\max }
\end{gathered}
$$

where Excess $=$ Excess energy, $\max _{R E}=$ The maximum value of real excess energy reached

To maximize profits, producer saves in a list all requests received and it may periodically decide to propose to sell a surplus of energy (which is not required). It sends to all neighbors that they have requested for energy for the present time slot a sale request by proposing a low price. If there are any responses, it decides to sell the surplus of energy to grid. The proposed approach encourages the cooperation between microgrids to ensure their profits which consist of increasing their profitability.

\section{Performance Analysis And Simulation}

The main idea of our proposed RTDEM consists in improving the microgrids behavior in order to increase their profitability. As we can see, there are several operations (e.g. buying, selling, making decision, etc.) that are executing simultaneously and in different location (i.e. microgrids) and potentially interacting with each other. This may lead to concurrent operations and to competition between microgrids who are trying to reach their own goal. For this, Java concurrency (multi-threading) has been used in order to develop a simulator and validate then the proposed energy management approach. It allows also multiple microgrids to process multiple transactions in parallel and in real-time.

In order to prove the effectiveness of our solution, we evaluate in this section, during one year, the performance of our proposed RTDEM approach compared to:

- MAS, where a distributed energy resource management approach target to match the buyers and sellers in energy market [20],

iHEM, where individual microgrids (homes) harvest and consume energy by themselves without energy sharing [11].

For this evaluation, we consider a smart power distribution system which is composed of five microgrids $\left(M G_{1}, M G_{2}\right.$, $M G_{3}, M G_{4}$, and $M G_{5}$ ). For the purpose of study, each microgrid is selected to have between 1 to 15 users (homes, buildings, institutional buildings, etc.), and distributed renew- able sources. Each microgrid $n\left(M G_{n}\right)$ has its own profile. During a year, three types of profiles can exist:

Profile 1: the microgrid consumes, on average, the same quantity of energy as it produces:

$$
\mathrm{E}\left(P_{n}\right)-\mathrm{E}\left(C_{n}\right)=0
$$

with $P_{n}=$ production of microgrid $n, C_{n}=$ consumption of $M G_{n}, \mathrm{E}=$ mathematical expectation.

Profile 2: the microgrid consumes, on average, less than it produces:

$$
\mathrm{E}\left(P_{n}\right)-\mathrm{E}\left(C_{n}\right)>0
$$

Profile 3: the microgrid consumes, on average, more than it produces:

$$
\mathrm{E}\left(P_{n}\right)-\mathrm{E}\left(C_{n}\right)<0
$$

We assume that $M G_{1}, M G_{2}, M G_{3}, M G_{4}$, and $M G_{5}$ have, respectively, Profile 3, 3, 1, 2 and 2. Table I present the profile of each microgrid. For this evaluation, we consider that $\mathrm{E}\left(C_{n}\right)$ is roughly equal to 3 kilowatts $(\mathrm{kW})$, ie. every $M G_{n}$ consumes on average roughly $3 \mathrm{~kW}$.

\begin{tabular}{|c|c|}
\multicolumn{2}{|c|}{ TABLE I: Microgrid's PROFILE } \\
\hline microgrid & Profile \\
\hline microgrid 1 $\left(M G_{1}\right)$ & $\mathrm{E}\left(P_{1}\right)=1 / 3 \mathrm{E}\left(C_{1}\right)$ \\
\hline microgrid 2 $\left(M G_{2}\right)$ & $\mathrm{E}\left(P_{2}\right)=2 / 3 \mathrm{E}\left(C_{2}\right)$ \\
\hline microgrid 3 $\left(M G_{3}\right)$ & $\mathrm{E}\left(P_{3}\right)=\mathrm{E}\left(C_{3}\right)$ \\
\hline microgrid 4 $\left(M G_{4}\right)$ & $\mathrm{E}\left(P_{4}\right)=4 / 3 \mathrm{E}\left(C_{4}\right)$ \\
\hline microgrid 5 $\left(M G_{5}\right)$ & $\mathrm{E}\left(P_{5}\right)=5 / 3 \mathrm{E}\left(C_{5}\right)$ \\
\hline
\end{tabular}

We assume that BP/unit $=\$ 0.21$ and $\mathrm{SP} /$ unit $=\$ 0.5$, with unit $=100$ Watts $(\mathrm{W})=0.1$ kilowatts $(\mathrm{kW})$.

We assume that the cost generation of each microgrids depends on its profile (the one who produces more has a greater cost). The value of the unit cost of energy (ARL) 
affects MAS. If ARL $\leq \mathrm{BP}+\epsilon$, in MAS, seller will earn almost nothing. Else (if ARL > SP), in MAS, seller may not sell anything to its neighbors. For this reason, we considered we considered an ideal case for MAS in which $\mathrm{ARLMG}_{1}=\mathrm{BP}+\epsilon=2.3, A R L_{M G_{2}}=2.95, A R L_{M G_{3}}=3.6$, $A R L_{M G_{4}}=4.25, A R L_{M G_{5}}=\mathrm{SP}-\epsilon=4.9$. However, high producer like $M G_{5}$ cannot increase its profitability when the supply is greater than the demand, because it proposes a high selling price.

At first, as no sales have been made yet, every $M G_{n}$ initializes the profitability factor on its estimation at $50 \%$.

Contrary to assumption considered by [20], we assume a realistic hypothesis where the demand and supply are not remaining constant during an interval/time slot.

In the following we present 3 scenarios where the consumption and production, for each $M G_{n}$, are assumed varying according to three main distributions: constant, uniform and normal (or Gaussian). In each figure (except for "Real expense" results and "Microgrids' profile"), the yellow color represents all the operations with neighbors, the green color represents the amount of energy consumed locally (from local energy sources), and the red color represents all the operations with grid (utility companies).

\section{A. Scenario 1: Constant Consumption and Production}

In order to underline the basic solutions to behavior, this first scenario compares our solution with MAS [20] and iHEM [11] in a simple case where we suppose that the consumption and production of energy are not changing over the time (in each time slot). We assume that a microgrid requires the same quantity of energy $3 \mathrm{~kW}$. In this case, sellers will make always profitable sales, their profitability factor will be equal to $100 \%$, because advance estimation may give a certain input. Fig. 1 shows the quantity of energy purchased, for each $M G_{n}$, from local energy sources (green color), neighbors (yellow color) and from grid (red color). Fig. 2 shows the expense of the quantity purchased from neighbors and from grid. Fig. 3 shows the quantity of energy supplied, for each microgrid, to neighbors and to grid. Fig. 4 shows the gain of the quantity supplied.
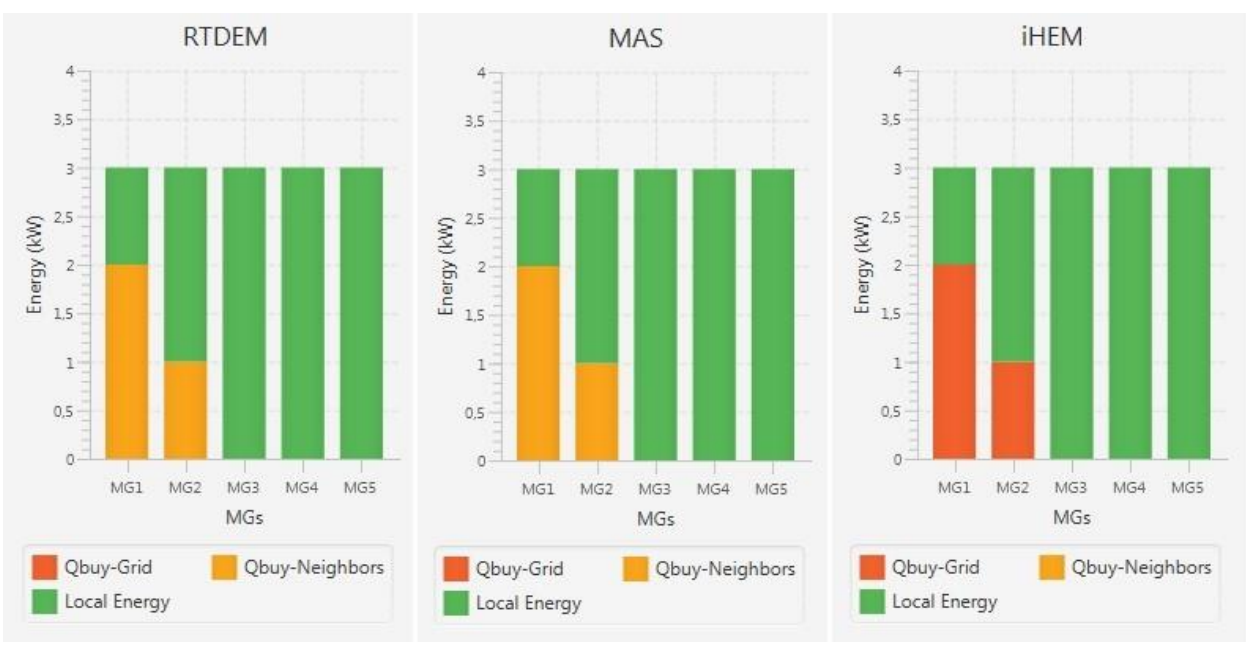

Fig. 1. Scenario1-energy purchased.
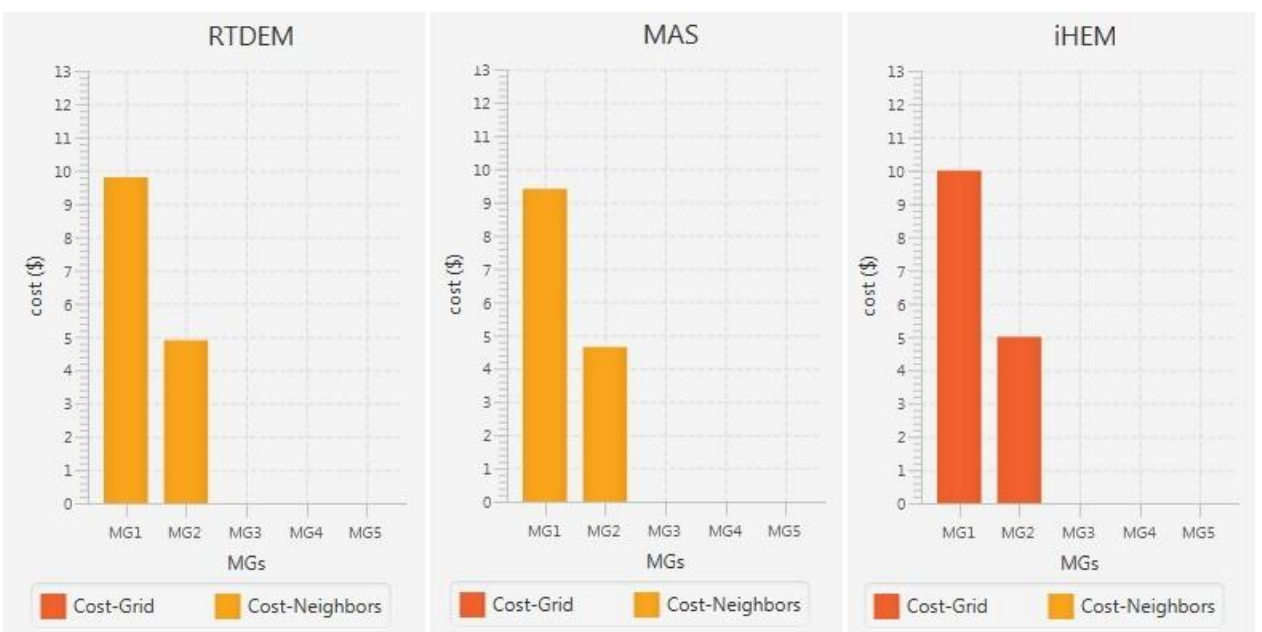

Fig. 2. Scenario1-Expense.

Simulation results show that $M G_{3}, M G_{4}$ and $M G_{5}$ satisfy their needs from their local energy (Fig. 1) and they don't need to buy energy from neighbors or utility companies (grid). They expense $\$ 0$ (see Fig. 2). On the contrary, $M G_{1}$ and $M G_{2}$ are not able to locally satisfy their needs (see Fig. 1). With iHEM, they will request the missing only from grid since this solution does not consider neighboring exchange (see Fig. 1). On contrary, with RTDEM and MAS, they will independently request the missing energy of $M G_{4}$ and $M G_{5}$ (see Fig. 1). RTDEM and MAS decrease slightly ${ }^{1}$ the energy

${ }^{1}$ In this case, sellers propose a high selling price because the demand is equal than the supply. 
cost compared to iHEM by benefitting from neighborhood energy surplus (see Fig. 2). With RTDEM and MAS, $M G_{4}$ and $M G_{5}$ supply their excess to their neighbors (see Fig. 3). However, with iHEM, this excess is supplied to grid (see Fig. 3). Moreover, $M G_{4}$ and $M G_{5}$ in RTDEM earn more than in MAS and iHEM. This conclusion is true in all cases except in the particular case when $\mathrm{ARL}=(\mathrm{SP}-\epsilon)$ in which RTDEM $=$ MAS, like $M G_{5}$ which is in its ideal case (Fig. 4). Indeed, with RTDEM, microgrids make more profit by selling their energy surplus at a higher price (thanks to our selling price function) while, with MAS, microgrids accept any offer greater than or equal to their ARL.

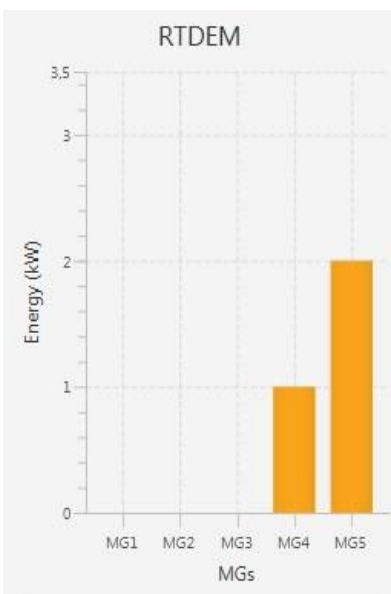

Qsell-Grid Qsell-Neighbors

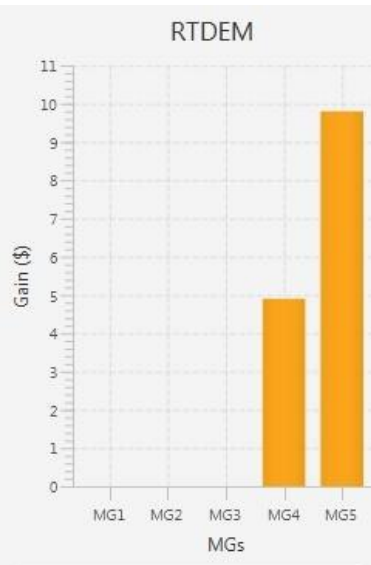

Gain-Grid

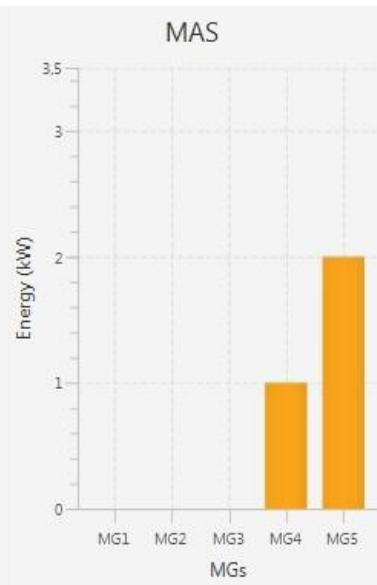

Qsell-Grid

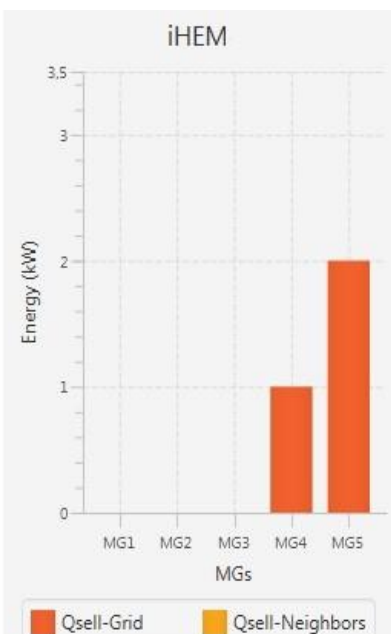

Qsell-Grid Qsell-Neighbors

Fig. 3. Scenario1-Quantity sold.

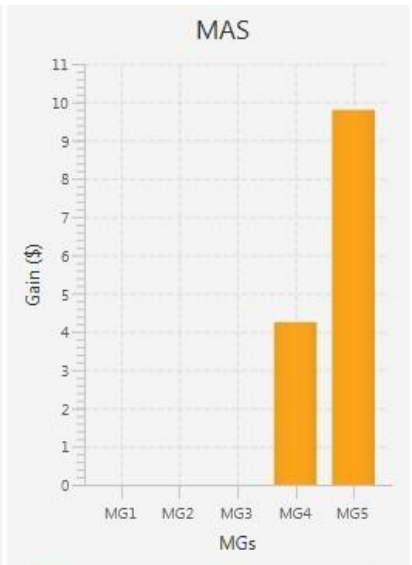

Gain-Grid Gain-Neighbors

Fig. 4. Scenario1-Gain.

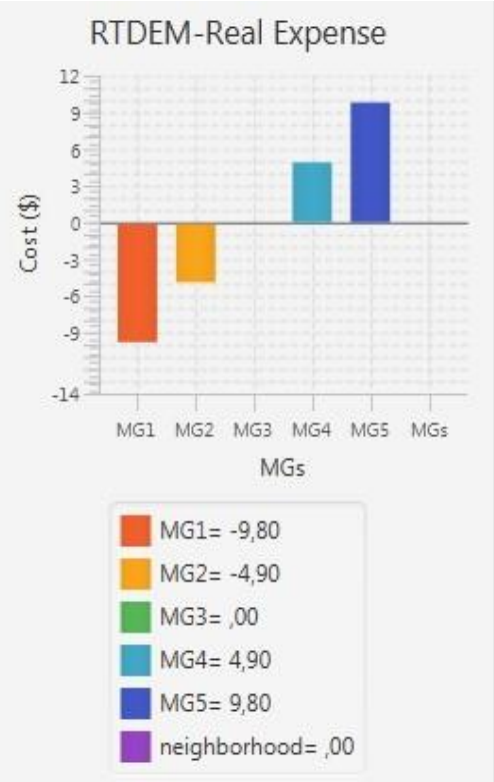

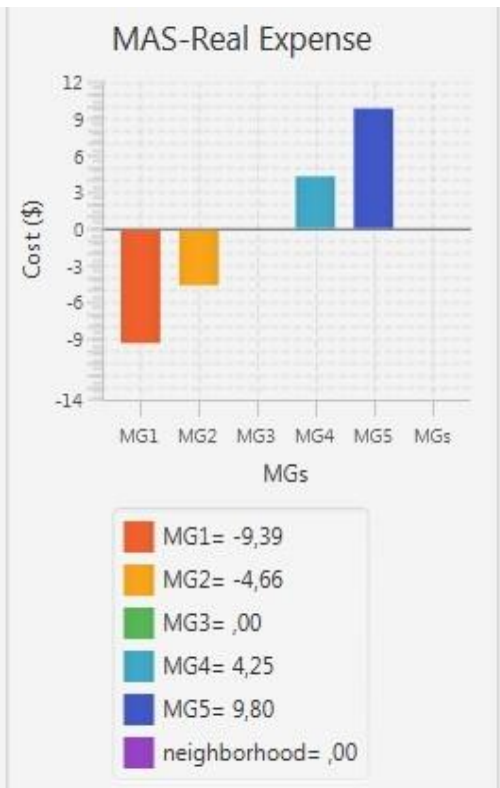

Fig. 5. Scenario1-Real expense.
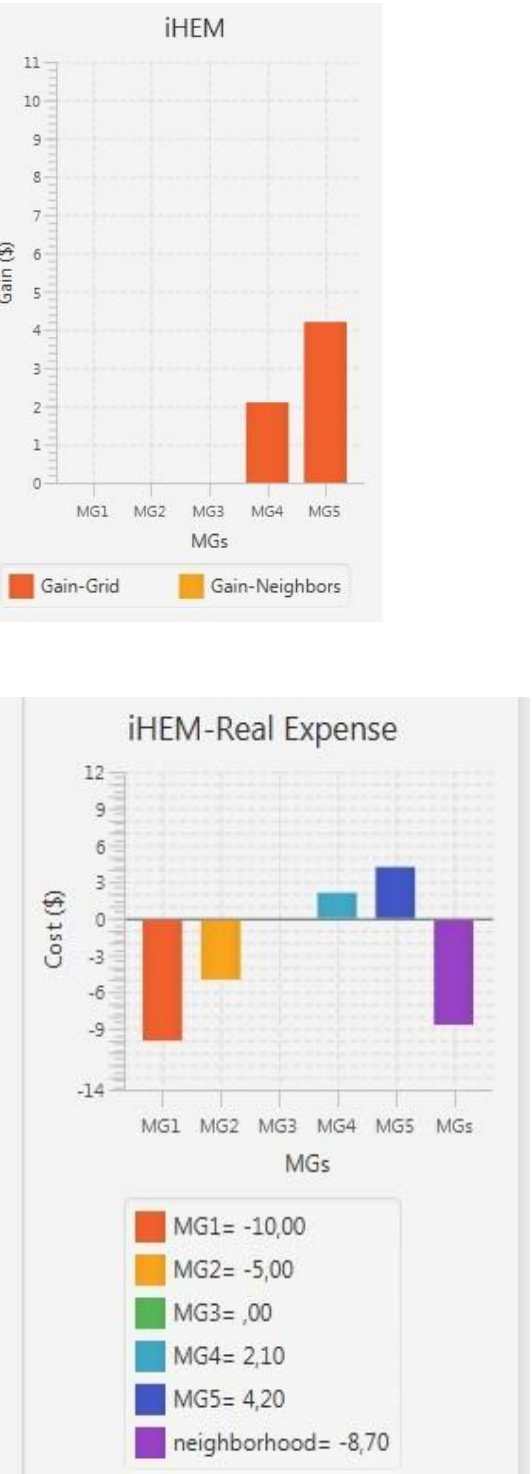
Fig. 5 presents, on average, the Real Expense $R E_{n}{ }^{2}$ for each $M G_{n}$ and for the neighborhood ( $R E_{\text {neighborhood }}=$ $\left.\sum R E_{n}\right)$.

If $R E_{n}$ is positive then the $M G_{n}$ managed to satisfy its need, and its expense (Expense ${ }_{n}$ ) is equal to \$0. Else (if $R E_{n}$ is negative) then the microgrid $\mathrm{n}$ must expense $\$\left|R E_{n}\right|$.

Likewise, if REneighborhood is positive then the neighborhood managed to satisfy its need, and its expense (REneighborhood) is equal to \$0. Else (if RENeighborhood is negative) then neighborhood must expense $\$ \mid$ RENeighborhood|. The sign determines if it is a benefice $(\geq$ $0)$ or a deficit $(<0)$. In RTDEM and MAS, Expenseneighborhood $=\$ 0$. However, in iHEM, Expense neighborhood $=\$ \mid$ REneighborhood $\mid=\$ 8.7$.

Simulation of MAS gives the same results of real expense as RTDEM, because in constant case electricity use and generation is stable and certain. MAS is in its ideal case, the demand and supply remain constant during an interval, which is not realistic. For this case, the profitability factor does not affect energy selling price because sellers make all time a good sales based on their certain estimation for the requested interval/time slot. In fact, we notice that the profitability factor of $M G_{1}, M G_{2}$ and $M G_{3}$ remain equal to $50 \%$ (initial value), because they never made sales. The profitability factor of $M G_{4}$ and $M G_{5}$ are equal to $100 \%$, because they make always a profitable sale.

\section{B. Scenario 2: Variable Production and Consumption (Uniform Distribution)}

We consider more realistic assumption where the consumption and production of energy for each requested time slot are not constant (stable) but may vary with the weather, as changes in temperature and humidity affect the demand. With this realistic assumption a profitability factor is necessary to avoid all non-optimal sales, because the estimation of energy production and consumption may give an uncertain input. Profitability factor determines the microgrid's trust on its estimation in order to propose a very profitable price.

For this case, we assume that $\mathrm{Cn} \in[0 \mathrm{~kW}, 6 \mathrm{~kW}]$ for each MGn. As production depends on the consumption then: $P_{1}$ $\in[0 \mathrm{~kW}, 2 \mathrm{~kW}], \mathrm{P} 2 \in[0 \mathrm{~kW}, 4 \mathrm{~kW}], \mathrm{P} 3 \in[0 \mathrm{~kW}, 6 \mathrm{~kW}]$, $\mathrm{P} 4 \in[0 \mathrm{~kW}, 8 \mathrm{~kW}], \mathrm{P} 5 \in[0 \mathrm{KW}, 10 \mathrm{~kW}$ ] (see previous table), Fig. 6 shows the average, during one year, of energy consumption and production for each microgrid $\mathrm{n}$.

Simulation results show that MAS requests more quantity of energy than real-time solution (RTDEM and iHEM), because it satisfy each microgrid's demand based on an estimation of energy consumption and generation which can be greater, equal or lower to microgrid's real consumption (see Fig. 7). In fact, sometimes the actual energy consumption may exceed or not the predicted consumption. Then, when the (real consumption $<$ predicted consumption), microgrids in MAS may buy energy at a higher price which will not be required later. In this case, they will be forced to sell it to the grid at a lower price (i.e. at BP). Contrary to MAS, RTDEM and iHEM real-time manage the energy demand. RTDEM and iHEM request the same quantity of

${ }^{2}$ With REn $=$ Gainn - Expensen. (See Fig. 5 and Fig. 7). energy because they satisfy in real time each microgrid's demand (see Fig. 7). RTDEM uses more efficient local renewable energy than MAS and iHEM. Note that for a microgrid, it is always more profitable to use its own energy then the energy from the neighbors and lastly the energy from the grid. We also observe that RTDEM use less energy from grid than MAS and iHEM (see Fig. 7). Consequently, with RTDEM microgrids expense less to grid (see Fig. 8) because they require less energy from network since they use more efficient renewable energy.

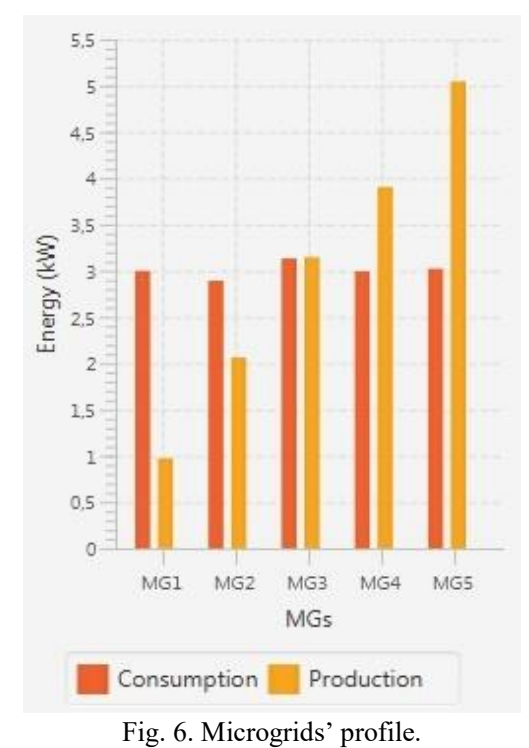

Indeed, with MAS, a $M G_{n}$ periodically estimate the surplus of energy in order to sell it. However, due to the nature of the $M G_{n}$ consumption and production that vary over the time, sometimes, the real energy consumption is greater than the estimation or the real energy production is lower than the estimation. This induces a lack of local energy and forces the $M G_{n}$ to buy energy at a price potentially higher than the energy it had sold before. Likewise, when the (real surplus $<$ predicted surplus), they may sell a surplus (at lower price) which will be required later. Then, they must buy it from grid or neighbors at a higher price. While with RTDEM, microgrids sell their energy surplus at a flexible selling price which is profitable compared with MAS which has the price equal or greater to its ARL. On the other hand, iHEM sells its real energy surplus only to the grid (see Fig. 9).

RTDEM sells less energy to grid and more efficient energy to neighbors than iHEM and MAS (see Fig. 9). Consequently, RTDEM earns more from neighbors and less from grid compared to MAS and iHEM (see Fig. 10). Since RTDEM is real time based, local energy is not sold while required in a short term based on our defined profitability factor. In other words, with RTDEM, the local renewable energy produced is more profitable and will be promoted better than MAS and iHEM (thanks to our energy selling price which is based on the profitability factor and the excess energy). Based on this, RTDEM proposes a selling energy price adapted to each seller, which is not the case in the others approaches.

Fig. 11 shows the profitability factor for each $M G_{n}$ on its estimation. The 1 st $\mathrm{MG}\left(M G_{1}\right)$ is a smaller producer and during 365 days he made any sales due to the stagnation 
that we can see in the curve. In Fig. 11, we observe that the profitability factor of $M G_{2}$ is very low because it sells its excess which is required later. The profitability factor of the 3nd $\mathrm{MG}\left(\mathrm{MG}_{3}\right)$ is greater than the one of the 2 st $\mathrm{MG}$, because he took better decisions regarding the sales made. The profitability factor of the 1st, 2 nd and $3 \mathrm{rd} \mathrm{MG}$ is lower compared to the one of the 4th and 5th MG because the first 3
MGs $\left(M G_{1}, M G_{2}\right.$ and $\left.M G_{3}\right)$ are smaller producers than the 4th and 5th MG and when they sell their excess then they will have a very high possibility to need their estimated excess. Based on the profitability factor that it is able to specify the trust on the estimation, RTDEM proposes a selling price adapted to each seller, which is very profitable compared to the others approaches.
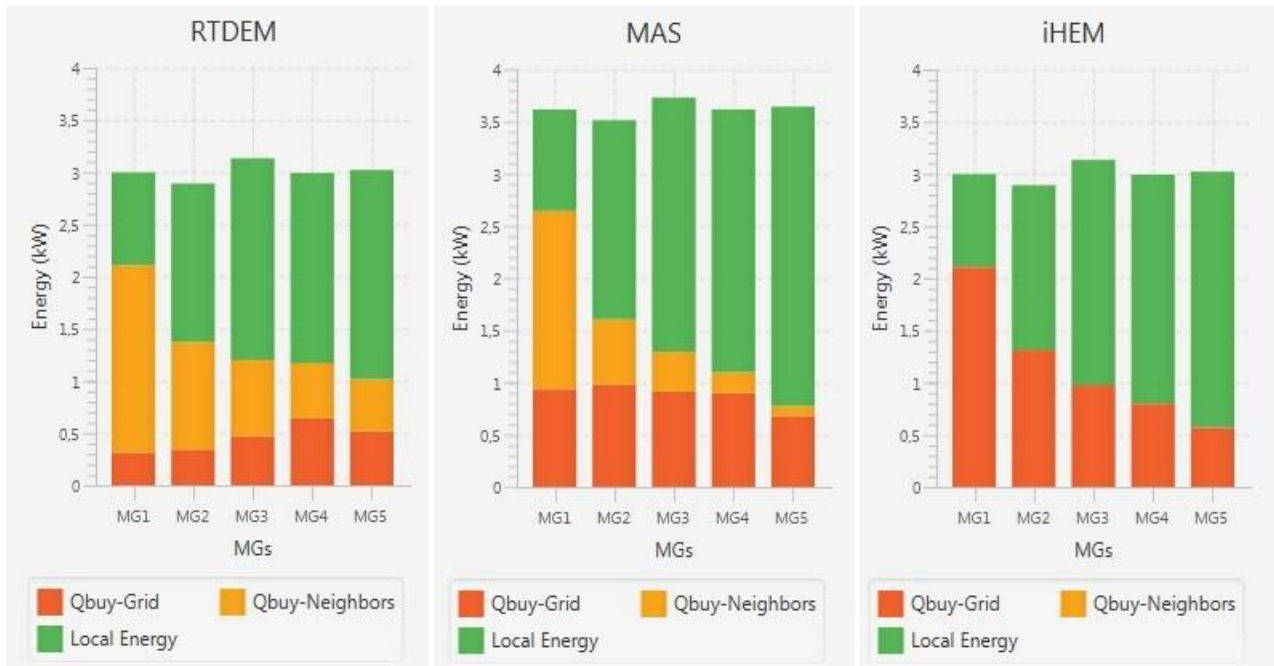

Fig. 7. Scenario2-Energy source.
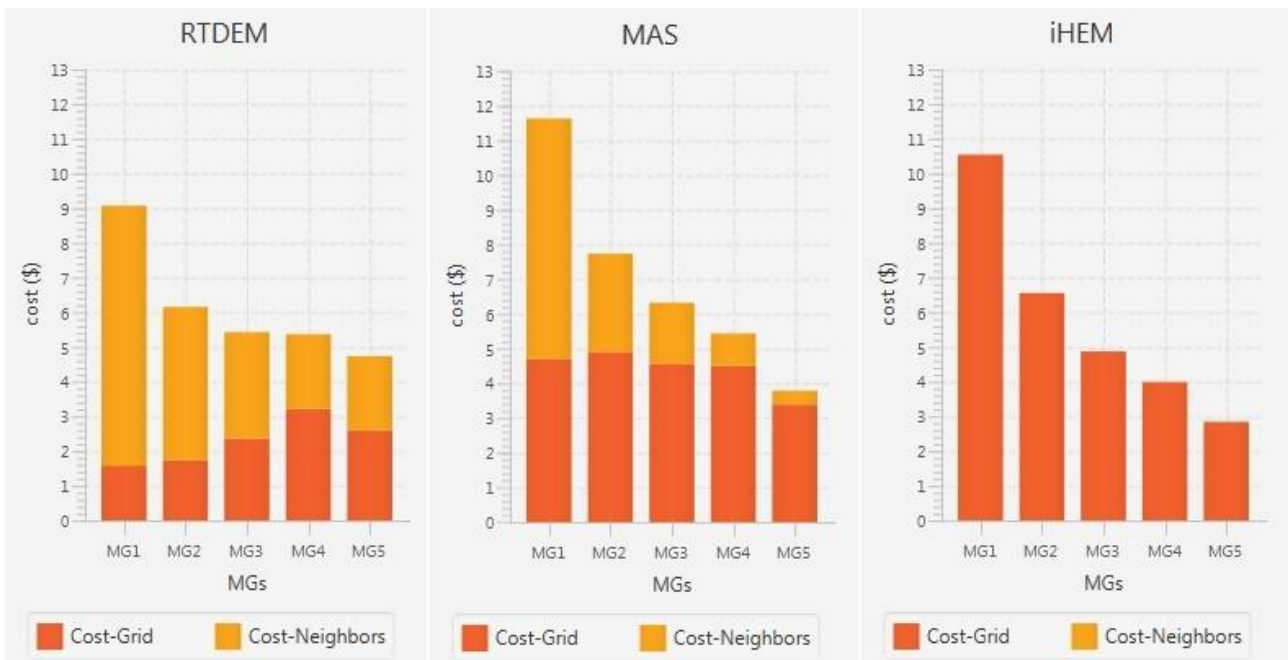

Fig. 8. Scenario2-expense.
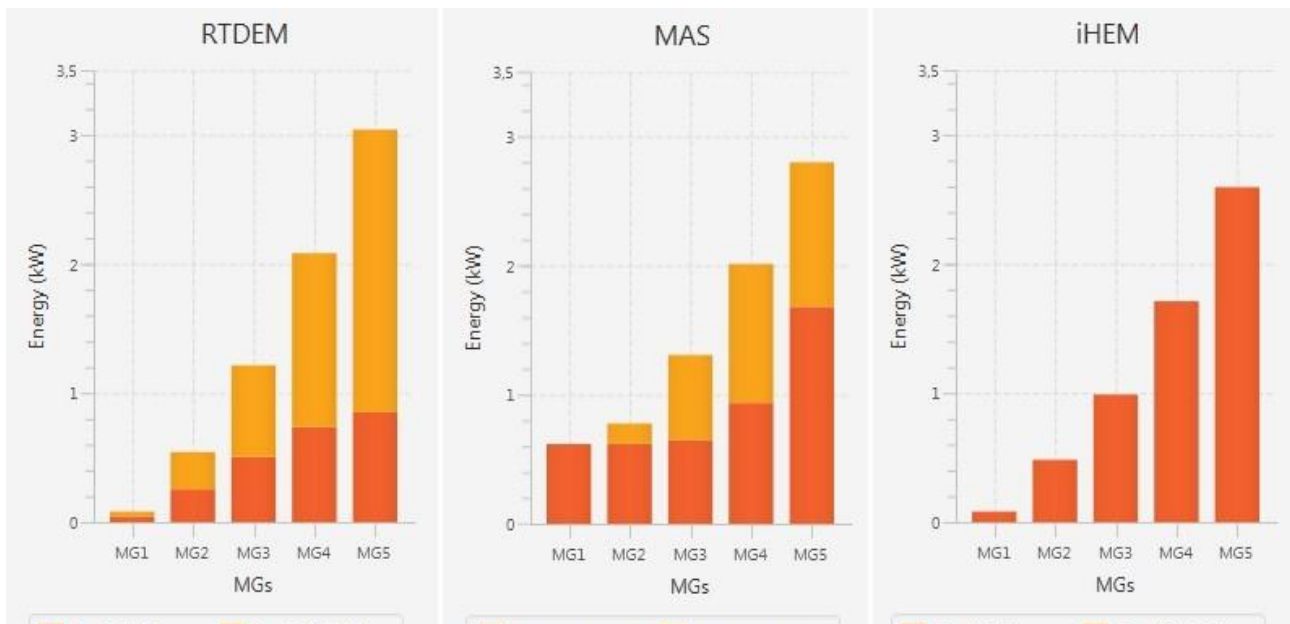

Qsell-Grid Qsell-Neighbors

Fig. 9. Scenario2-quantity sold. 

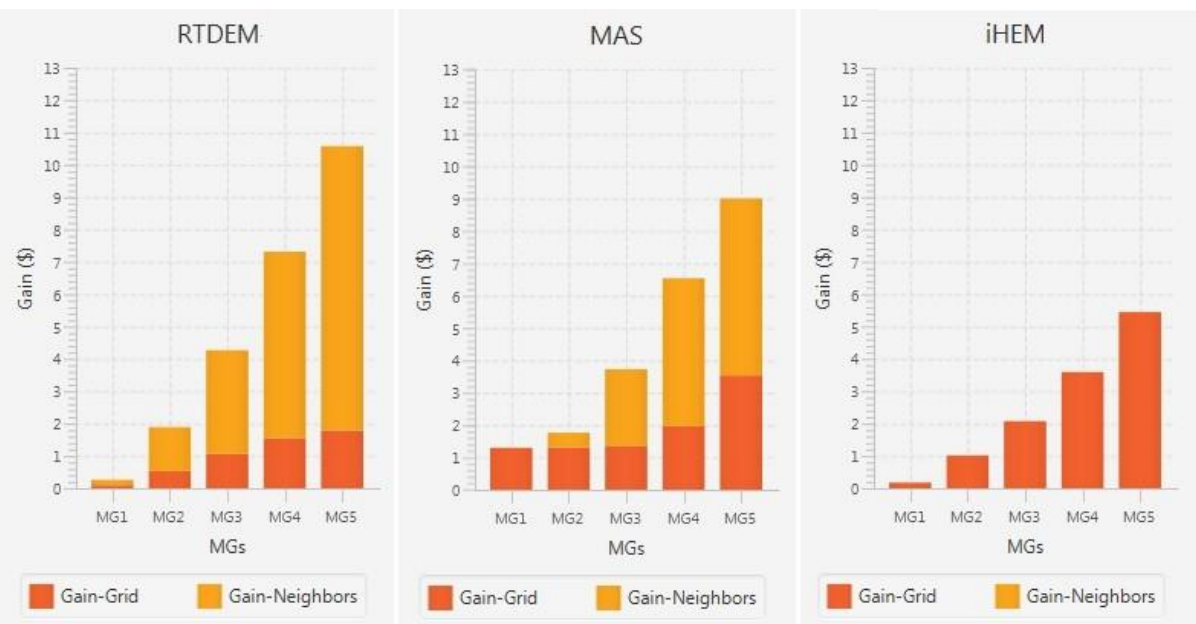

Fig. 10. Scenario2-Gain.
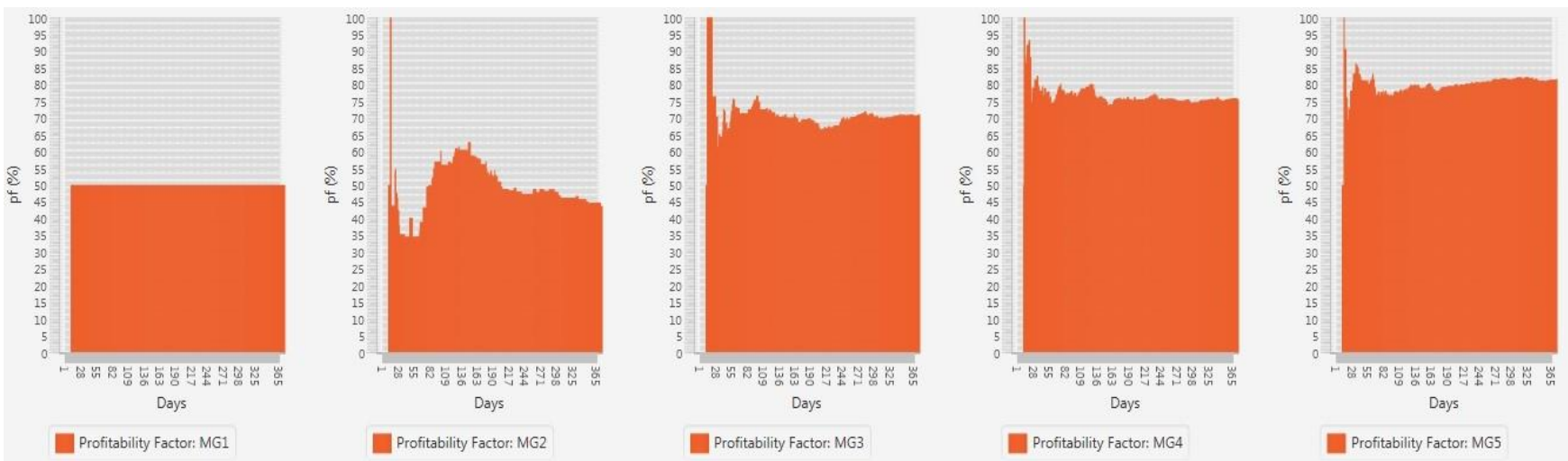

Fig. 11. Scenario2-Profitability factor of each microgrid.

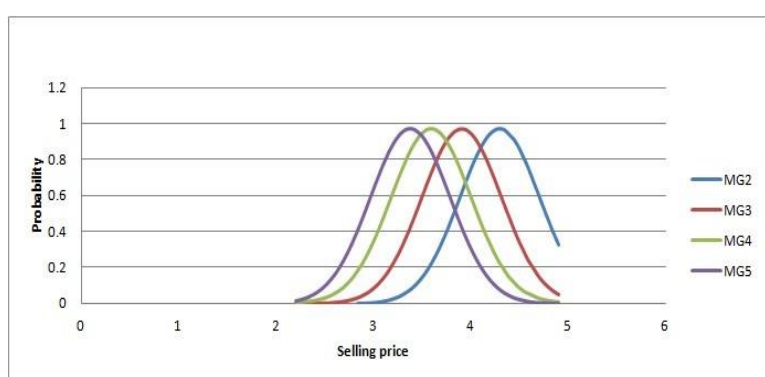

Fig. 12. Scenario2-Normal distribution showing proposed energy selling price.

With RTDEM, high producer proposes, when the supply is greater than the demand, a low energy selling price contrary to small producer which propose a high price. As $M G_{1}, M G_{2}$ and $M G_{3}$ are small producers then, in RTDEM, their proposed energy selling price is high compared to high producer $\left(M G_{4}, M G_{5}\right)$ which propose a less selling price in order to sell a maximum of energy. Contrary to MAS, the energy selling price depends on the ARL which is less for $M G_{1}, M G_{2}$ and $M G_{3}$. Based on this, small producer expense less with RTDEM compared to MAS because in the majority of cases they satisfy their needs from higher producers. Fig. 12 shows the normal distributions to make detailed of the proposed price for each microgrid in a short time scale (two week). In Fig. 12, we show four normal distributions having the same standard deviation (0.5). The purple (left-most) distribution, for $M G_{5}$, has a mean of 3.37, the distribution in red, for $M G_{4}$, has a mean of 3.59 , the distribution in red, for $M G_{3}$, has a mean of 3.90 , and the distribution in blue (right-most), for $M G_{2}$, has a mean of 4.29 .
iHEM and RTDEM use a real time decision that may give a stable (or certain) consumption, which causes a transition to a highly efficient economy. However, energy exchange between neighbors must also be considered (like MAS and RTDEM). Fig. 13 shows that $M G_{1}, M G_{2}$ and $M G_{3}$ expense less with RTDEM than with MAS and IHEM. $M G_{4}$ and $M G_{5}$ expense $\$ 0$ in RTDEM, iHEM and MAS. As their $\left(M G_{4}\right.$ and $M G_{5}$ ) sign is positive, then they have a benefice (gain). The benefice in RTDEM is greater than in MAS and iHEM. Our proposal combine Real Time decision and neighboring energy trading which guarantee more profitability for microgrids than MAS and iHEM (Expense neighborhood $=$ $\$ 6.45$ in RTDEM, $=\$ 12.59$ in MAS, $=\$ 16.50$ in iHEM (Fig. 13)).

We have verified the efficiency of our approach by varying the interval of consumption:

- Case 1: $C_{n} \in[2.7,3.3]$ for each $M G_{n}, P_{1} \in[0.9$, 1.1], $P_{2} \in[1.8,2.2], P_{3}[2.7,3.3], P_{4} \in[3.6,4.4], P_{5} \in$ $[4.5,5.5]$

- Case 2: $C_{n} \in[2.4,3.6]$ for each $M G_{n}, P_{1} \in[0.8$, 1.2], $P_{2} \in[1.6,2.4], P_{3} \quad[2.4,3.6], P_{4} \in[3.2,4.8], P_{5} \in$ $[4,6]$

- Case 3: $C_{n} \in[1.5,4.5]$ for each $M G_{n}, P_{1} \in[0.5$, 1.5], $P_{2} \in[1,3], P_{3}[1.5,4.5], P_{4} \in[2,6], P_{5} \in[2.5,7.5]$ - Case 4: $C_{n} \in[0,6]$ for each $M G_{n}, P_{1} \in[0,2], P_{2}$ $\in[0,4], P_{3}[0,6], P_{4} \in[0,8], P_{5} \in[0,10]$

- Case 5: $C_{n} \in[0,9]$ for each $M G_{n}, P_{1} \in[0,3], P_{2} \in$ $[0,6], P_{3}[0,9], P_{4} \in[0,15], P_{5} \in[0,15]$ 

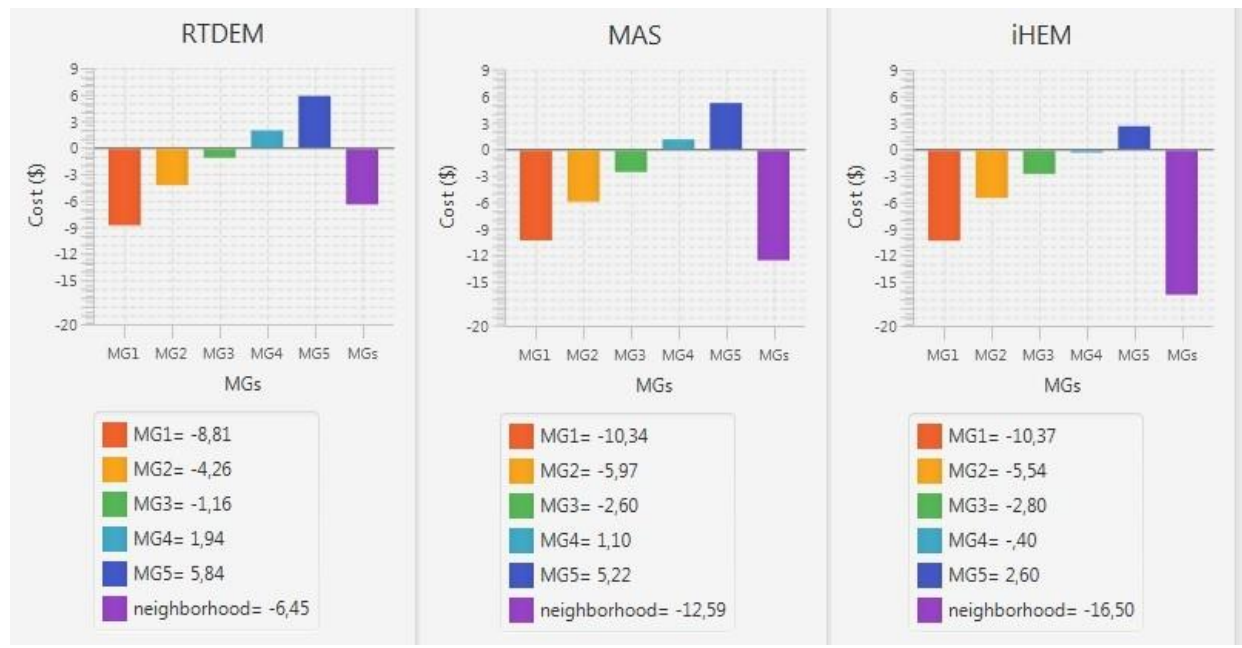

Fig. 13. Scenario2-Real expense.

We conclude that when the interval is large, neighborhood in RTDEM spends much less compared to MAS and iHEM that when the interval is small (Fig. 14).

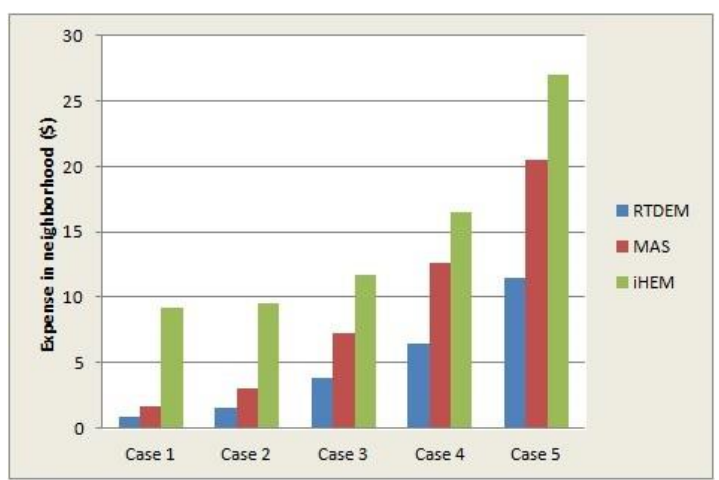

Fig. 14. Scenario2-Real expense when varying the interval of consumption.

\section{Scenario 3: Variable Production and Consumption (Normal Distriution)}

In our third case, we consider a more realistic assumption where the consumption and production of energy vary over time according to normal distribution. Normal distribution depends on two factors: the mean and the standard deviation. The mean of the distribution determines the location of the center of the graph, and the standard deviation determines the height of the graph. For consumption, we assume that the mean is equal to 3 and the deviation is equal to 1 . As production depends on consumption, we adapted the mean and deviation, for each producer, to keep its profile.

Simulation results (see Fig. 15) show that $M G_{1}, M G_{2}$ and $M G_{3}$ expense less with RTDEM than with MAS and iHEM. $M G_{4}$, and $M G_{5}$ expense $\$ 0$ with RTDEM, MAS and iHEM. whereas, $M G_{4}$, and $M G_{5}$ earn more with RTDEM than with MAS and iHEM. RTDEM decreases expenses in neighborhood in comparison with MAS and iHEM (see Fig. 15). Expense $_{\text {Neighborhood }}=\$ 3.80$ in RTDEM, $\$ 7.37$ in MAS, $\$ 12.75$ in iHEM.
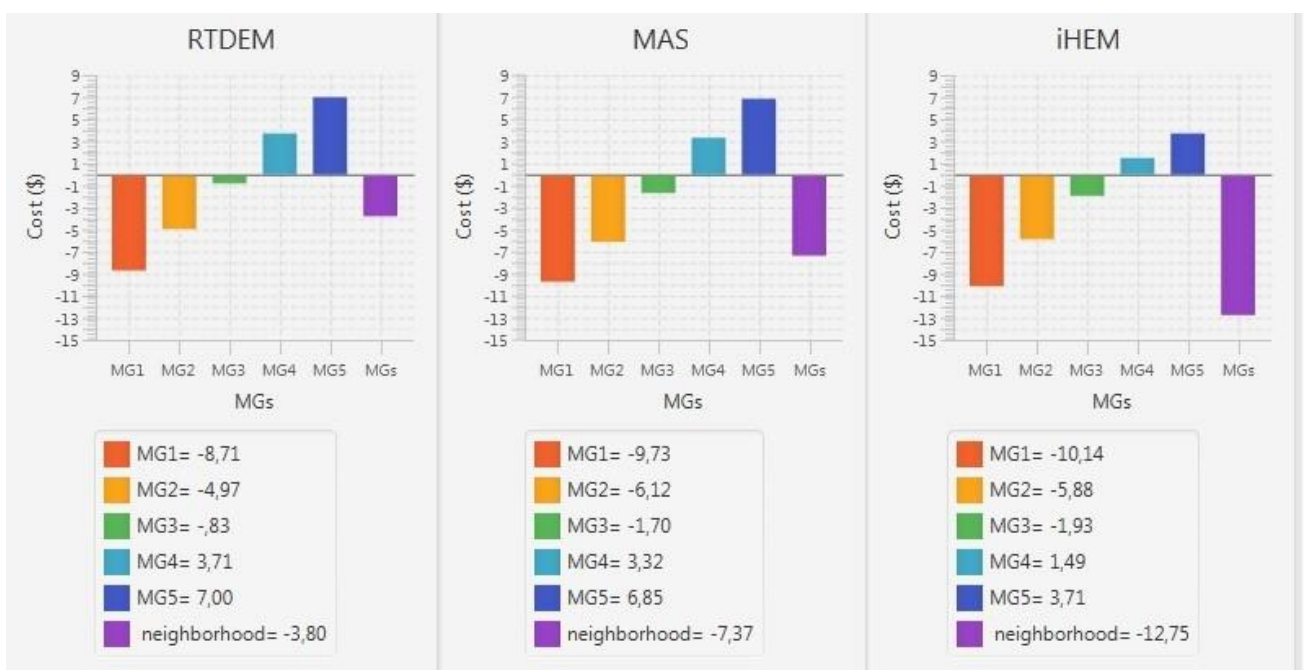

Fig. 15. Scenario3-Real expense.

We have verified the efficiency of our approach using other value of the standard deviation. We notice that when the standard deviation is large, RTDEM spends much less compared to MAS (it deceives sorely and therefore these results are not as effective) and iHEM when the standard deviation is small.
Real-time manages the energy demand and share energy in neighborhood cause a transition to a highly efficient economy. The encouragement of the investment of renewable energy leads to reduce our need from fossil fuels. The efficient use of renewable energy sources is the only way that degradation of Earth's climate system can be halted, and 
the only real option for raising severe consequences on the habitats [22].

\section{CONCLUSION}

In this paper, we have proposed a Real-Time Distributed Energy Management (RTDEM) to share renewable excess energy among microgrids. RTDEM is based on a profitability factor that a seller defines on its estimated excess energy in order to realize optimal sales. The profitability factor is defined based on the microgrid's trust on its estimation. Furthermore, RTDEM is able to determine the best alternative to satisfy microgrid's requirements (i.e. decrease energy costs and maximize energy gains). When the microgrid is in buyer mode, the best alternative is determined by solving an optimization problem considering different criteria such as selling price, available energy, etc. It decreases its energy bill by using its own energy then the energy from the neighbors lastly the energy from the grid. Whereas, when the microgrid is in seller mode, the best alternative is determined by proposing an optimal energy selling price. We presented simulation results and assessed the performance of our proposed algorithm, results proving the effectiveness of our solution compared with other approaches thanks to our adequately and flexible energy selling price. Simulation results show that RTDEM improves the energy sharing efficiency that leads to reducing gas emissions and encouraging the investment of renewable energy by minimizing energy cost and maximizing gain based on the interactions with microgrids without need to shift their demand. Thus, a profit is made even when considered loads are not able to be shifted, which is not the case for the similar existing works.

\section{REFERENCES}

[1] M. Hook and T. Xu, "Depletion of fossil fuels and anthropogenic climate change-a review," Energy Policy, vol. 52, pp. 797-809, 2013.

[2] Breakdown of Electricity Generation by Energy Source. [Online]. Available:

http://www.tsp-data-portal.org/Breakdown-of-Electricity-Generationby-Energy-Source

[3] A. Dai, "Increasing drought under global warming in observations and models," Nat Clim Change, vol. 3, no. 52-58, 2012.

[4] D. Shindell et al., "Simultaneously mitigating near-term climate change and improving human health and food security," Science, vol. 335, pp. 183-189, 2012.

[5] X. Fang, S. Misra, G. Xue, and D. Yang, Smart Grid - The New and Improved Power Grid: A Survey, 2011.

[6] R. Walawalkar, S. Fernands, N. Thakur, and K. R. Chevva, "Evolution and current status of demand response (DR) in electricity markets: Insight from PJM and NYISO," Energy, vol. 35, pp. 1553-1560, 2010.

[7] W.-H. Liu, K. Liu, and D. Pearson, "Consumer-centric smart grid," Innovative Smart Grid Technologies, pp. 1-6, 2011.

[8] K. Herter, "Residential implementation of critical-peak pricing of electricity," Energy Policy, vol. 35, pp. 2121-2130, 2007.

[9] C. Triki and A. Violi, "Dynamic pricing of electricity in retail markets," Q. J. Oper. Res., vol. 7, pp. 21-36, 2009.

[10] P. Centolella, "The integration of price responsive demand into regional transmission organization (RTO) wholesale power markets and system operations," Energy, vol. 35, pp. 1568-1574, 2010.

[11] M. Erol-Kantarci and H. T. Mouftah, "Wireless sensor networks for cost-efficient residential energy management in the smart grid," IEEE Trans. Smart Grid, vol. 2, pp. 314-325, 2011.

[12] A.-H. Mohsenian-Rad and A. Leon-Garcia, "Optimal residential load control with price prediction in real-time electricity pricing environments," IEEE Trans. Smart Grid, vol. 1, pp. 120-133, 2010.

[13] M. A. A. Pedrasa et al., "Coordinated scheduling of residential distributed energy resources to optimize smart home energy services," IEEE Trans. Smart Grid, vol. 1, pp. 134-143, 2010.
[14] E.-K. Melike and T. M. Hussein, "Wireless sensor networks for domes- tic energy management in smart grids," IEEE, pp. 63-66, 2010.

[15] J. M. Lujano-Rojas et al., "Optimum residential load management strategy for real time pricing (rtp) demand response programs," Energy Policy, vol. 45, pp. 671-679, 2012.

[16] A.-H. Mohsenian-Rad et al. "Optimal and autonomous incentive-based energy consumption scheduling algorithm for smart grid," in Proc. IEEE Conf. Innovative Smart Grid Technol, 2010, pp. 1-6.

[17] M. Erol-Kantarci et al., "TOU-aware energy management and wireless sensor networks for reducing peak load in smart grids," in Proc. Green Wireless Commun. Networks Workshop, Fall 2010, pp. 1-5.

[18] A. Molderink, V. Bakker, M. B. Johann, L. Hurink, and G. J. M. Smit, "Management and control of domestic smart grid technology," IEEE Trans. Smart Grid, vol. 1, pp. 109-119, 2010.

[19] W. Zhong, Z. Huang, T. Zhu, Y. Gu, Q. Zhang, P. Yi, D. Jiang, S. Xiao, "IDES: Incentive-driven distributed energy sharing in sustainable microgrids," in Proc. IGC, 2014, pp. 1-10.

[20] H. S. V. S. K. Nunna and S. Doolla, "Multiagent-based distributed-energy-resource management for intelligent microgrids," IEEE Trans. Ind. Electron, vol. 60, pp. 1678-1687, 2013.

[21] I. Khan, N. Javaid, M. N. Ullah, A. Mahmood, and M. U. Farooq, "A survey of home energy management systems in future smart grid communications." in Proc. 2013 International Conf. on Broadband and Wireless Computing, Commun. and Appli., 2013, pp. 459-464.

[22] Worldwatch. [Online]. http://www.worldwatch.org/node/6428

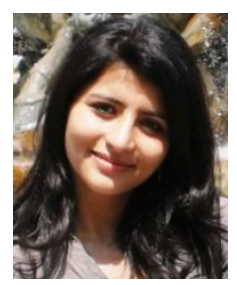

Zeineb Mhadhbi received the master degree in computer science from the University of Tunis, El Manar, Faculty of Sciences of Tunis, in 2012. She is currently working toward the Ph.D. degree in the Faculty of Sciences of Tunis. Her research interests are smart microgrids, multiagent system applications, and demand-side management.

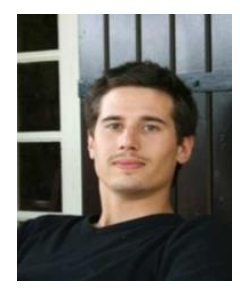

Cedric Gueguen is an associate professor at University of Rennes 1 and member of ATNET team at IRISA Labs. Its main research interests are related to wireless network and particularly to scheduling algorithms and green networking. In 2010, he was researcher (ATER) in the department "Protocols Architecture \& Software for Networks" (PASNET) at the University Paris-Est Marne-la-Vallée (LIGM). In November 2010, he obtained his $\mathrm{PhD}$ from the University Pierre et Marie Curie while working in the Networks and Performance nalysis group at the aboratory LIP6. Its PhD thesis focused on the efficient support of multimedia services in OFDM wireless networks, both in the uplink and in the downlink. New access schemes were studied which further increases the benefits of opportunistic scheduling mainly by extending this cross layer technique to higher layers. Before, he hold a M.Sc. in Networking from University Pierre et Marie Curie (UPMC), with a major in performance evaluations.

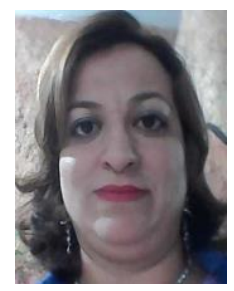

Sajeh Zairi is currently an associate professor at the ESEN (Univ. Manouba-Tunisia) and researcher at the Mediatron laboratory (University of Carthage, Tunisia). She was graduated with a Computer Science Engineer degree at FST (Univ. Tunis El ManarTunisia), followed by a Joint $\mathrm{PhD}$ in Computer Science and Automation at FST and INSA-Lyon (France). Her main research interest relates to the formal specification and verification of discrete event systems, the optimization of distributed systems, the configuration of sensor network, and the power management in sensor networks and smart grid.

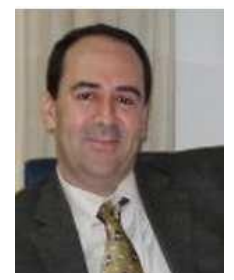

Belhassen Zouari is a professor in computer science at the Higher School of Communications Sup'Commember of Mediatron Lab-University of Carthage, Tunisia. His activities include research works in the fields of verification of automated systems and security issues. He worked on formal verification methods based on coloured Petri nets and applied the related techniques in various fields as Flexible Manufacturing Systems, Wireless Sensor Networks and Business Process Management. He obtained a Doctorate (French PhD) from the University of Paris6 (1993) in Computer Science, and a "Habilitation Universitaire" from the University of Tunis (2005). 